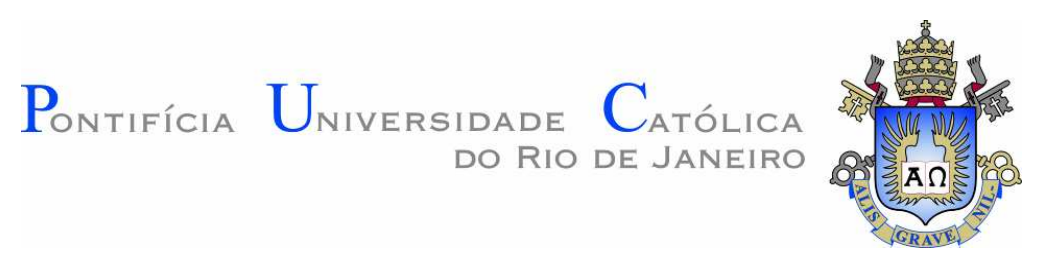

Ana Gabriela Pilla zur Nedden

\title{
Análise do processo de quebra de gotas de uma emulsão óleo-água no escoamento entre discos paralelos
}

Dissertação de Mestrado

Dissertação apresentada como requisito parcial para obtenção do título de Mestre pelo Programa de PósGraduação em Engenharia Mecânica da PUC-Rio.

Orientador: Prof. Marcio da Silveira Carvalho

Co-Orientador: Prof. Valdir Estevam

Rio de Janeiro

Setembro de 2010 


\section{Análise do processo de quebra de gotas de uma emulsão óleo-água no escoamento entre discos paralelos}

Dissertação apresentada como requisito parcial para obtenção do grau de Mestre pelo Programa de PósGraduação em Engenharia Mecânica do Centro Técnico Científico da PUC-Rio. Aprovada pela Comissão Examinadora abaixo assinada.

Prof. Marcio da Silveira Carvalho Orientador Departamento de Engenharia Mecânica - PUC-Rio

Prof. Valdir Estevam

Co-Orientador PETROBRAS

Prof. Luis Fernando Alzuguir Azevedo Departamento de Engenharia Mecânica - PUC-Rio

Prof. Geraldo Afonso Spinelli Martins Ribeiro PETROBRAS

Prof. José Eugenio Leal Coordenador Setorial do Centro Técnico Científico - PUC-Rio 
Todos os direitos reservados. É proibida a reprodução total ou parcial do trabalho sem autorização da universidade, da autora e do orientador.

\section{Ana Gabriela Pilla zur Nedden}

Bacharel em Engenharia Mecânica pela Universidade Federal do Rio de Janeiro, em 2004. Especialização em Engenharia de Petróleo pela Universidade Federal da Bahia, em 2006 e em Engenharia de Dutos pela PUC-Rio, em 2007.

Ficha Catalográfica

Nedden, Ana Gabriela Pilla zur
Análise do processo de quebra de gotas de uma
emulsão óleo-água no escoamento entre discos paralelos /
Ana Gabriela Pilla zur Nedden ; orientador: Marcio da
Silveira Carvalho ; co- orientador: Valdir Estevam. - 2010.
89 f. : il. (color.) ; 30 cm
Dissertação (mestrado)-Pontifícia Universidade
Mecânica, 2010.
Inclui bibliografia
1. Engenharia mecânica - Teses. 2. Emulsão. 3.
Quebra de gotas. 4. Petróleo. I. Carvalho, Marcio da
Silveira. II. Estevam, Valdir. III. Pontifícia Universidade
Católica do Rio de Janeiro. Departamento de Engenharia
Mecânica. IV. Título.


Dedico este trabalho à minha família. 


\section{Agradecimentos}

Ao Professor Luiz Fernando sem o qual este mestrado não teria começado;

À PUC pela bolsa em grande parte do curso;

À Petrobras pela oportunidade concedida, em especial Geraldo Spinelli e Caetano Frisolli;

Ao Carlos Dias, do CENPES, responsável pelo projeto e execução do dispositivo experimental, sem o qual este trabalho não seria possível;

Aos amigos da Petrobras que assumiram minhas tarefas durante o período final, em especial à Denise, Fabiano e Sérgio Paulo

Ao Professor Márcio Carvalho pela orientação e incentivo essenciais para a realização deste trabalho;

Ao co-orientador Valdir Estevam por toda ajuda e atenção despendida.

À Ranena pelas inúmeras ajudas no laboratório;

Aos colegas do laboratório Danmer, Raúl, Oswaldo, Alessandro e Martin que tornaram o mestrado mais agradável

À todos aqueles que, direta ou indiretamente, colaboraram para que este trabalho consiga atingir aos objetivos propostos. 


\section{Resumo}

zur Nedden, Ana Gabriela Pilla; Carvalho, Márcio da Silveira. Análise do processo de quebra de gotas de uma emulsão óleo-água no escoamento entre discos paralelos. Rio de Janeiro, 2010. 89 p. Dissertação de Mestrado - Departamento de Engenharia Mecânica, Pontifícia Universidade Católica do Rio de Janeiro.

A produção de petróleo é geralmente acompanhada pela produção de água, que pode estar presente sob forma livre e emulsionada. As emulsões podem ser encontradas em quase todas as fases de produção: nos reservatórios, nos poços produtores, nas facilidades de produção, nos dutos de transporte, no processamento e no armazenamento O sistema de Bombeio Centrifugo Submerso (BCS) é um dos métodos utilizados na indústria para a elevação de petróleo para campos localizados tanto em terra como em mar. Ainda que seja o método de elevação artificial mais apropriado para a produção de petróleo quando há elevada produção de água, a eficiência da bomba é bastante reduzida quando há manuseio de misturas de água e óleo e a formação de emulsões.Este trabalho teve como objetivo uma primeira contribuição ao estudo do cisalhamento aos quais os fluidos estão expostos durante a passagem por um estágio de uma Bomba Centrífuga Submersa e seus efeitos no grau de emulsificação da mistura de água e óleo. Foi construído um aparato experimental composto por dois discos, um giratório e um fixo. Ao escoar através destes a mistura de fluidos sofre deformação cisalhante e extensional e seu grau de emulsificação, isto é, tamanho médio e distribuição dos diâmetros de bolhas, é medido a montante e a jusante do dispositivo.

\section{Palavras-chave}

Emulsão; quebra de gotas; petróleo. 


\section{Abstract}

Pilla zur Nedden, Ana Gabriela; Carvalho, Márcio da Silveira (Advisor). An analysis of the drop breakup process in an oil in water emulsion flowing between parallel disks Rio de Janeiro, 2010. 89 p. MSc. Dissertation Departamento de Engenharia Mecânica, Pontifícia Universidade Católica do Rio de Janeiro.

Oil production is usually associated with the production of water, which may be found as a continuous phase or as a dispersed phase, in emulsions. Emulsions may be found in almost all the production stages: in reservoirs, production wells, production facilities, pipelines, in processing and in storage. The Electric Submersible Pump (ESP) is one of the methods employed in the industry to lift oil from fields located on land as well as at sea. Though this is the most appropriate artificial lift method for oil production when a considerable amount of water is produced, a pump's efficiency is a greatly reduced when mixtures of water and oil are handled and emulsions begin to form. This paper intends to study the shearing imposed to fluids when flowing through a stage in a Electric Submersible Pump, and its effects in the degree of emulsification of the water and oil mixture. An experimental device was assembled consisting in two disks, one that spins and the other fixed. When flowing through them, a mixture of fluids is subjected to shearing and extensional deformation, and its degree of emulsification, i.e.: the average size and the bubble diameter distribution, may be measured upstream and downstream from the device.

\section{Keywords}

Emulsion, drop breakup, shearing rate. 


\section{Sumário}

1 Introdução

2 Revisão Bibliográfica $\quad 20$

2.1. O problema da água produzida. 20

2.2. Bombeio Centrífugo Submerso 21

2.3. Emulsões 24

2.4. Surfactantes 27

2.5. O processo de emulsificação 29

2.6. Estabilidade das emulsões 30

2.7. Principais fatores que afetam a estabilização das emulsões de petróleo 32

2.8. Quebra de emulsões: 34

2.9. Viscosidade das emulsões: 37

2.10. Mecanismos de quebra de gotas em uma emulsão: 39

2.10.1. Mecanismo de quebra de gota por cisalhamento 39

2.11. Estudos sobre quebra de gotas em uma emulsão 40

3 Estudo Experimental $\quad 44$

3.1. Descrição da bancada experimental 44

3.2. Preparação da Emulsão $\quad 47$

3.3. Procedimento experimental 50

3.4. Matriz de Testes 53

3.5. Incertezas experimentais $\quad 55$

4 Resultados e discussões $\quad 57$

4.1. Análise Geral das Medidas de Distribuição de Tamanho de Gotas: $\quad 57$

4.2. Dados Medidos 58

4.3. Parâmetros adimensionais estudados para análise dos dados medidos: 61

$\begin{array}{ll}\text { 4.4. Resultados } & 67\end{array}$ 
5 Conclusões e recomendações 78

5.1. Conclusões 78

5.2. Recomendações $\quad 79$

$\begin{array}{ll}\text { Referências } & 81\end{array}$

Apêndice A Exemplo de uma análise do Mastersizer 84

Apêndice B Cálculo de Incertezas $\quad 85$

Apêndice C Desenho técnico do dispositivo construído 89 


\section{Lista de figuras}

Figura 2-1 - Desenho simplificado de um estágio de uma BCS composto de um impelidor e um difusor. 22

Figura 2-2 - Principais componentes do conjunto de BCS. 23

Figura 2-3 - Desenho esquemático dos três tipos de emulsões: óleo em água, água em óleo e água em óleo em água.

Figura 2-4 - Arranjos tridimensionais de esferas. (Adaptado de Becher, 2001)

Figura 2-5 - Forças atuando nas moléculas na interface e no interior de dois líquidos. (Adaptado de Becher, 2001)

Figura 2-6 - Redução da tensão superficial com a concentração de surfactante na fase contíunua e definição de Concentração Micelar Crítica (CMC). (Adaptado de Schramm, 1992)

Figura 2-7 - Formação de micelas com a adição de surfactante acima da CMC. (Adaptado de Schramm, 1992)

Figura 2-9 - (a) cargas em torno da interface formando a dupla camada e (b) repulsão eletrostática resultante (Adaptado de Cunha, 2007).

Figura 2-10 - Perfis de temperatura em um poço de produção em mar e em um poço de produção em terra.

Figura 2-11 - Processos de Espessamento, Sedimentação, Floculação e Coalescência (Adaptado de Coutinho, 2005).

Figura 2-12 - Variação da viscosidade com $\phi$. (Adaptado de Karcher, 2008)

Figura 2-13 Mecanismo quebra de gota por cisalhamento. (Adaptado de Olbricht, 1996)

Figura 2-14 - Mecanismo quebra de gota por cisalhamento. (Adaptado de Olbricht, 1996)

Figura 3-1 - Esquema gráfico da Bancada Experimental.

Figura 3-2 - Dispositivo construído para realização do experimento.

Figura 3-3 Trajetória do escoamento no interior do dispositivo.

Figura 3-4 - Em detalhe, o anel espaçador entre os discos. 
Figura 3-5 - Mistura de água e surfactante na preparação da fase contínua da emulsão.

Figura 3-6 - Detecção de diâmetros maiores, que indicava entrada de gás no sistema (formação de bolhas).

Figura 3-7 - Mastersizer 2000E, equipamento utilizado para as medições dos diâmetros das gotas.

Figura 4-1 - Distribuição dos diâmetros de gotas na entrada e na saída do dispositivo.

Figura 4-2 - Diâmetros de entrada em função da ordem de execução das medições.

Figura 4-3 - perfil de velocidades no escoamento laminar em placas paralelas.

Figura 4-4 - $D_{s} / D_{e}$ medidos e seus respectivos tempos de residência no interior do dispositivo experimental.

Figura 4-5 - $D_{s} / D_{e}$ em função de suas taxas de cisalhamento.

Figura 4-6 - Resultados de $D_{s} / D_{e}$ em função dos respectivos números de Reynolds.

Figura 4-8 $D_{s} / D_{e}$ em função do Número de Weber.

Figura 4-9 Valores de $D_{s} / D_{e}$ e os respectivos números de Froude. $\quad 74$

Figura 4-10 - $D_{s}$ em função do número de Reynolds. $\quad 75$

Figura 4-11 - $D_{s}$ em função do número de Weber. $\quad 75$

Figura 4-12 - $D_{s} / D$ em função do número de Weber do dispositivo. 76

Figura 4-13 - Valores de $D_{s}$ em função do número de Froude calculado para a gota. 


\section{Lista de tabelas}

Tabela 3-1 Propriedades físico-químicas da fase contínua. 49

Tabela 3-2 Propriedades físico-químicas da fase dispersa (óleo). 49

Tabela 3-3 Matriz de teste. $\quad 54$

Tabela 3-4 - Valores calculados para as propriedades físico-químicas da emulsão e suas respectivas incertezas.

Tabela 3-5 Incertezas percentuais calculadas. 56

Tabela 4-1 - Valores de $D_{s}, D_{e}$ medidos e $D_{s} / D_{e}$ calculado para cada ponto experimental. $\quad 59$

Tabela 4-2 - Tempo de residência dos fluidos no interior do dispositivo $\begin{array}{ll}\text { experimental. } & 67\end{array}$

Tabela 4-3 Número de Reynolds, antes da entrada do fluido no dispositivo experimental, calculado para as três vazões estudadas. 68

Tabela 4-4 - Número de Reynolds de acordo com as condições experimentais indica escoamento laminar também no interior dos discos. $\quad 69$

Tabela 4-5 Taxas de cisalhamento quando consideramos apenas o movimento de translação. $\quad 70$

Tabela 4-6 Taxas de cisalhamento aplicadas ao escoamento considerando apenas o movimento de rotação. $\quad 70$

Tabela B-1 - Incertezas na medida do espaçamento entre os discos. 85

Tabela B-2 - Incertezas nas medidas de vazão. 85

Tabela B-3 - Incertezas nas medições da rotação do disco interno do dispositivo. $\quad 85$

Tabela B-4 - Incertezas nas medições dos diâmetros das gotas. 86

Tabela B-5 Incertezas percentuais calculadas. 88 


\section{Nomenclatura}

\section{Letras Latinas}

$A: \quad$ área $\left[\mathrm{L}^{2}\right]$;

$d$ : diâmetro das gotas [L];

$d_{\text {máx }}: \quad$ diâmetro máximo estável em determinadas condições [L];

D: $\quad$ diâmetro do disco interno do dispositivo experimental [L];

$D_{50}$ : diâmetro médio medido ma análise de distribuição de tamanho de gotas feita pelo Mastersizer [L];

$D_{e}$ : $\quad$ diâmetro da gota medida a montante do dispositivo (diâmetro de entrada) [L];

$D_{h}$ : $\quad$ diâmetro hidráulico do dispositivo experimental [1];

$D_{\text {mangueira }}$ : diâmetro da mangueira [L];

$D_{s}$ : $\quad$ diâmetro da gota medida a jusante do dispositivo (diâmetro de saída) [L];

$D_{s} / D_{e}: \quad$ razão entre os diâmetros médios (D50) medidos a montante e a jusante do dispositivo[1];

$\mathrm{Ca}: \quad \quad \quad$ Número de capilaridade [1];

Fr: $\quad$ número de Froude [1];

$F r_{g}: \quad$ número de Froude da gota [1];

$g$ : $\quad$ aceleração da gravidade $\left[\mathrm{LT}^{-2}\right]$;

$h$ : $\quad$ espaçamento entre os discos do dispositivo experimental [L];

$L$ : $\quad$ comprimento [L];

$N: \quad$ rotação do dispositivo $\left[\mathrm{T}^{-1}\right]$;

$n$ : $\quad$ número de medidas de uma amostra feitas pelo Mastersizer. [1];

$P: \quad$ perímetro [L];

$Q: \quad$ vazão $\left[\mathrm{L}^{3} \mathrm{~T}^{-1}\right]$;

$\bar{R}$ : $\quad$ raio médio do disco interno do dispositivo experimental [L];

$R: \quad$ raio dispositivo experimental [L]; 
$R_{i}$ : $\quad$ raio inicial do dispositivo experimental por onde ocorre a entrada de fluidos [L];

$R_{f}$ : $\quad$ raio final do dispositivo experimental por onde ocorre a saída de fluidos [L];

Re : $\quad$ número de Reynolds [1];

$\operatorname{Re}_{g}: \quad$ número de Reynolds da gota[1];

$\operatorname{Re}_{\text {mangueira }}$ : número de Reynolds na entrada do dispositivo [1];

$\operatorname{Re}_{\text {escoamento }}$ : número de Reynolds na entrada do dispositivo [1];

$t_{R}$ : $\quad$ tempo de residência dos fluidos no interior do dispositivo [T];

$u: \quad$ velocidade $\left[\mathrm{LT}^{-1}\right]$;

$u_{s}: \quad$ velocidade de sedimentação $\left[\mathrm{LT}^{-1}\right]$;

$\Delta u: \quad$ diferença de velocidades nas duas extremidades do diâmetro da gota $\left[\mathrm{LT}^{-1}\right]$;

$U_{\text {med }}$ : velocidade transversal média dos fluidos no interior do dispositivo $\left[\mathrm{LT}^{-1}\right]$;

$U_{\text {mangueira }}$ : velocidade do escoamento na mangueira $\left[\mathrm{LT}^{-1}\right]$;

$U_{\text {máx }}$ : velocidade transversal máxima dos fluidos no interior do dispositivo $\left[\mathrm{LT}^{-1}\right]$;

$\quad U W: \quad$ velocidade dos fluidos no interior do dispositivo $\left[\mathrm{LT}^{-1}\right]$;

$V: \quad \quad \quad \quad$ volume no interior do dispositivo [ $\left.\mathrm{L}^{3}\right]$;

$W_{\text {med }}$ : $\quad$ velocidade angular média dos fluidos no interior do dispositivo $\left[\mathrm{LT}^{-1}\right]$;

We : $\quad$ número de Weber [1];

We $e_{\text {crit }}: \quad$ número de Weber crítico[1];

$W e_{g}: \quad$ número de Weber da gota [1];

$W e_{T}: \quad$ número de Weber do tanque/ do dispositivo[1];

\section{Letras Gregas}

$\mu$ : $\quad$ viscosidade dinâmica da emulsão $\left[\mathrm{L}^{-1} \mathrm{MT}^{-1}\right]$;

$\mu_{\delta}: \quad$ viscosidade dinâmica do óleo $\left[\mathrm{L}^{-1} \mathrm{MT}^{-1}\right]$;

$\mu_{f c}: \quad$ viscosidade dinâmica da fase contínua $\left[\mathrm{L}^{-1} \mathrm{MT}^{-1}\right]$; 
$\rho: \quad$ massa específica da emulsão $\left[\mathrm{L}^{-3} \mathrm{M}\right]$;

$\rho_{\dot{\sigma}}: \quad$ massa específica do óleo $\left[\mathrm{L}^{-3} \mathrm{M}\right]$;

$\rho_{d}: \quad$ massa específica da fase dispersa $\left[\mathrm{L}^{-3} \mathrm{M}\right]$;

$\rho_{f c}: \quad$ massa específica da fase contínua $\left[\mathrm{L}^{-3} \mathrm{M}\right]$;

$\Delta \rho: \quad$ diferença entre as densidades das fases contínua e dispersa [L $\left.\mathrm{L}^{-3} \mathrm{M}\right]$;

$\omega: \quad \operatorname{rotação~}\left[\mathrm{T}^{-1}\right]$;

$v: \quad$ viscosidade cinemática $\left[\mathrm{L}^{2} \mathrm{~T}^{-2}\right]$;

$\dot{\gamma}_{\text {rotação }}: \quad$ taxa de cisalhamento devido ao movimento de rotação $\left[\mathrm{T}^{-1}\right]$;

$\dot{\gamma}_{\text {translação }}:$ taxa de cisalhamento devido ao movimento de translação $\left[\mathrm{T}^{-1}\right]$;

$\dot{\gamma}: \quad$ taxa de cisalhamento $\left[\mathrm{T}^{-1}\right]$;

$\sigma_{o}: \quad$ tensão superficial do óleo $\left[\mathrm{MT}^{-2}\right]$;

$\sigma_{f c}: \quad$ tensão superficial da fase contínua $\left[\mathrm{MT}^{-2}\right]$;

$\sigma: \quad$ tensão interfacial da emulsão $\left[\mathrm{MT}^{-2}\right]$;

$\phi$ : $\quad$ fração volumétrica da fase dispersa [1];

$\tau_{\mu c}: \quad$ força externa viscosa $\left[\mathrm{L}^{-1} \mathrm{MT}^{-2}\right]$;

$\tau_{\text {inercial }}: \quad$ força inercial $\left[\mathrm{L}^{-1} \mathrm{MT}^{-2}\right]$;

$\tau_{\text {int erfacial }}$ : força interfacial $\left[\mathrm{L}^{-1} \mathrm{MT}^{-2}\right]$;

\section{Siglas}

BCS: Bombeamento Centrífugo Submerso;

CMC: Concentração Micelar Crítica

DTG: Distribuição do diâmetro de gotas

A/O Emulsão de água em óleo

O/A: Emulsão de óleo em água 
"O essencial é invisível aos olhos" Saint-Exupéry 